
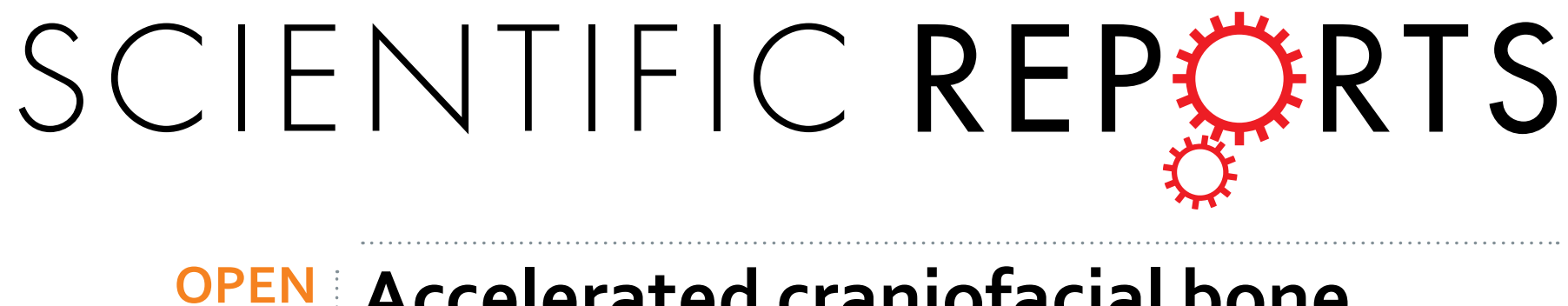

Accelerated craniofacial bone regeneration through dense collagen gel scaffolds seeded with

Received: 16 August 2016

Accepted: 14 November 2016

Published: 09 December 2016

\section{dental pulp stem cells}

Frédéric Chamieh ${ }^{1,2, *}$, Anne-Margaux Collignon ${ }^{1,3,{ }^{*}}$, Benjamin R. Coyac ${ }^{1,2}$, Julie Lesieur ${ }^{1}$, Sandy Ribes ${ }^{1}$, Jérémy Sadoine ${ }^{1}$, Annie Llorens ${ }^{1}$, Antonino Nicoletti ${ }^{4}$, Didier Letourneur ${ }^{4}$, Marie-Laure Colombier ${ }^{1,3}$, Showan N. Nazhat ${ }^{5}$, Philippe Bouchard ${ }^{1,2}$, Catherine Chaussain ${ }^{1,3}$ \& GaelY. Rochefort ${ }^{1}$

Therapies using mesenchymal stem cell (MSC) seeded scaffolds may be applicable to various fields of regenerative medicine, including craniomaxillofacial surgery. Plastic compression of collagen scaffolds seeded with MSC has been shown to enhance the osteogenic differentiation of MSC as it increases the collagen fibrillary density. The aim of the present study was to evaluate the osteogenic effects of dense collagen gel scaffolds seeded with mesenchymal dental pulp stem cells (DPSC) on bone regeneration in a rat critical-size calvarial defect model. Two symmetrical full-thickness defects were created ( $5 \mathrm{~mm}$ diameter) and filled with either a rat DPSC-containing dense collagen gel scaffold $(n=15)$, or an acellular scaffold $(n=15)$. Animals were imaged in vivo by microcomputer tomography (Micro-CT) once a week during 5 weeks, whereas some animals were sacrificed each week for histology and histomorphometry analysis. Bone mineral density and bone micro-architectural parameters were significantly increased when DPSC-seeded scaffolds were used. Histological and histomorphometrical data also revealed significant increases in fibrous connective and mineralized tissue volume when DPSC-seeded scaffolds were used, associated with expression of type I collagen, osteoblast-associated alkaline phosphatase and osteoclastic-related tartrate-resistant acid phosphatase. Results demonstrate the potential of DPSC-loaded-dense collagen gel scaffolds to benefit of bone healing process.

Tissue engineering approaches offer novel treatment modalities in numerous medical disciplines ${ }^{1}$, including bone augmentation procedures in oral surgery for dental implant placement and periodontal reconstructions. While autologous bone grafting is the current "gold standard" procedure in large defects ${ }^{2}$, it exhibits some limitations such as availability of sufficient graft volume, donor site morbidity or unpredictable bone resorption ${ }^{3,4}$. On the other hand, allografts or xenografts have been shown to be associated with immunoreactions, and a risk of transmission of either bacteria or viruses ${ }^{5,6}$. Although synthetic grafts can be easily absorbed, they do not exhibit osteoinductive properties. To overcome the drawbacks associated with grafting procedures, current bone tissue-engineering strategies are employing different combinations of osteoconductive substitutes, growth factors and stem/progenitor cells ${ }^{7}$. These biomaterial templates aim to not only provide an adequate volume for the prevention of soft tissue collapse into the intrabony defect, but also stimulate seeded cell migration, proliferation and differentiation through biological and mechanical cues.

${ }^{1}$ EA 2496 Orofacial pathologies, imaging and biotherapies, Dental School Faculty, University Paris Descartes, and Life imaging Platform (PIV), Montrouge, France. ${ }^{2}$ Department of Periodontology, Service of Odontology, Rothschild Hospital, AP-HP, University Denis Diderot, U.F.R. of Odontology, Paris, France. ${ }^{3}$ Department of Odontology, University Hospitals, AP-HP, Paris, France. ${ }^{4}$ INSERM U1148, Laboratory of Vascular Translational Science, University Paris Diderot, University Paris 13, Sorbonne Paris Cité, X Bichat Hospital, and Département Hospitalo-Universitaire (DHU) FIRE, F-75018 Paris, France. ${ }^{5}$ Department of Mining and Materials Engineering, McGill University, Montreal, Quebec, Canada. *These authors contributed equally to this work. Correspondence and requests for materials should be addressed to G.Y.R. (email: gael.rochefort@gmail.com) 
A large variety of biomaterials has been used as carriers in bone tissue engineering approaches, including the widely used three dimensional (3D) collagen-based biomimetic hydrogel scaffolds ${ }^{8,9}$. These hydrogel scaffolds are biocompatible, biodegradable with low antigenicity, which provide a favorable environment to support osteoblast attachment, proliferation, and differentiation ${ }^{10,11}$. Although collagen scaffolds are per se highly hydrated (with more than $95 \% \mathrm{w} / \mathrm{v}$ fluid) with weak mechanical properties for tissue replacement applications ${ }^{12}$, the "simple" plastic compression of the material rapidly increases the relative collagen fibrillar density (to more than $10 \%$ in weight) by removing the excess of fluid ${ }^{13}$. The "plastic compression" approach thus yields a type I collagen matrix with a fibrillar density similar to that of native bone matrix ${ }^{14-16}$. This process enables the rapid, controllable and reproducible production of dense collagen gel scaffolds with highly defined meso-structure and increased biomechanical properties, similar to that of the osteoid ${ }^{10,17,18}$. Furthermore, cell seeding constitutes part of the processing route, and the scaffolds provide the 3D structure for their growth and differentiation without compromising their viability ${ }^{13,19}$.

Mesenchymal stem cells (MSCs) are common candidates for scaffold-based tissue engineering ${ }^{20}$. Dental pulp stem cells (DPSCs) are neural crests derived cells ${ }^{21,22}$, which exhibit MSC characteristics and have the ability to differentiate into odontoblasts, adipocytes, osteoblasts, chondrocytes and myocytes ${ }^{23-26}$. Dental stem cells can be harvested from several dental sources, including DPSCs, stem cells from human exfoliated deciduous teeth (SHED), stem cells from the apical papilla (SCAP), periodontal ligament stem cells (PDLSC), and dental follicle precursor cells (DFSC) ${ }^{27}$. In particular, DPSCs are a potential alternative source for bone regeneration/healing and tissue engineering attributable to their high proliferation rates, their extended differentiation potential, and paracrine properties ${ }^{28-30}$. In addition, similarly to MSCs that have been shown to exhibit immunomodulatory properties in syngeneic, allogeneic and xenogeneic applications ${ }^{31}$, human DPSC transplanted into large rat calvarial defects have been demonstrated to differentiate into osteogenic cells without any graft rejection ${ }^{32}$. Thus, we hypothesized that dense collagen scaffold, which constitutes a physiological osteogenic extracellular matrix due to its elevated fibrillar density, combined with mesenchymal stem cells derived from the dental pulp, would enhance bone regeneration. Therefore, the aim of this study was to evaluate the osteogenic effects of dense collagen gel scaffolds seeded with rat DPSC (rDPSC) implanted in a rat critical-sized calvarial defect model. The bone repair process was dynamically monitored in vivo, using micro-computed tomography and analyzed by histomorphometry.

\section{Material and Methods}

Ethical approval and animal management. All experiments in this study were designed according to ARRIVE guidelines, and performed with a protocol approved by the Animal Care Committee of the University Paris Descartes (No. P2.JLS.174.10). Animals were maintained according to the guidelines for ethical conduct developed by the European Communities Council Directive (animal breeding agreement C92-049-01). All efforts were made to minimize their pain or discomfort.

WISTAR rats were purchased from Janvier Labs (Le Genest Saint Isle, France). They were housed at stable conditions $\left(22 \pm 2{ }^{\circ} \mathrm{C}\right)$ with a $12 \mathrm{~h}$ dark/light cycle, and with ad libitum access to water and food in the animal facility of the Department of Orofacial pathologies, imagery and biotherapies of Descartes University, Montrouge, France.

Isolation and culture of rat Dental Pulp Stem Cells. Multi-colony-derived rat dental pulp stem cells (rDPSCs) were obtained using a protocol adapted from Gronthos et al. from the molars of 4-day Wistar rats ${ }^{33}$. Under sterile conditions, rat molars were extracted and incubated at $4{ }^{\circ} \mathrm{C}$ for $45 \mathrm{~min}$ in phosphate-buffered saline (PBS) containing $100 \mathrm{U} / \mathrm{mL}$ penicillin/streptomycin (Gibco) and $250 \mu \mathrm{g} / \mathrm{mL}$ fungizone (Gibco), then in PBS containing $3 \mathrm{mg} / \mathrm{mL}$ type I collagenase (Worthington Biochem) and $2 \mathrm{U} / \mathrm{mL}$ dispase I (Roche) in a shaking incubator (at $37^{\circ} \mathrm{C}$ ) for $1 \mathrm{~h}$. The isolated cells were then plated on $0.1 \%$ gelatin-coated dishes in the Minimum Essential Media-alpha (Gibco) supplemented with $20 \% \mathrm{v} / \mathrm{v}$ fetal bovine serum (FBS) (Gibco) and $100 \mathrm{U} / \mathrm{mL}$ Penicillin/ streptomycin (Gibco), $1 \mathrm{ng} / \mathrm{ml} \mathrm{FGF-2} \mathrm{(Peprotech),} \mathrm{and} \mathrm{maintained} \mathrm{at} 37^{\circ} \mathrm{C}$ under $5 \% \mathrm{CO}_{2}$ atmosphere. The medium was changed after 2 days, and then twice a week. Upon reaching $70-80 \%$ confluence, cells were plated at $10^{4}$ cells per $\mathrm{cm}^{2}$. The medium was changed after 2 days, and then twice a week. The required cell number for the in vivo experiments was reached after $2-3$ passages.

Rat Dental Pulp Stem Cell Phenotype by Flow Cytometry. The expression of CD31 (AF488, \#MCA1334A488, BioRad, Oxford, UK), CD45 (PE, \#MCA43PE, BioRad), CD73 (eF450, \#48-0731-82, eBioscience Inc., San Diego, CA) and CD90 (PE-Cy5, \#ab95809, Abcam, Cambridge, UK) was analyzed by polychromatic flow cytometry (LSRII; Becton Dickinson, Franklin Lakes, NJ) with fluorochrome-conjugated monoclonal antibodies. Cells at passage 2 were detached by $4 \%$ lidocaine (Sigma, St Louis, MO). BD CompBeads particles (BD Biosciences) were used to calculate the compensation of fluorescence spillover. Only events from alive cells in both morphogate and singulet gates were analyzed.

Scaffold preparation. Plastically compressed collagen gels were used as 3D scaffolds and prepared as previously described ${ }^{10,13,34}$. The polymerizing type I collagen gel prepared solution (rat-tail tendon in $0.1 \% \mathrm{v} / \mathrm{v}$ acetic acid solution, neutralized by $\mathrm{NaOH}$ and obtained as previously described ${ }^{35}, 1.6 \mathrm{mg} / \mathrm{ml}$ final) acellular or with rDPSCs at a seeding density of $2 \times 10^{6} \mathrm{cell} / \mathrm{mL}$ was ice-cold mixed and platted into a 4 -well plate. After polymerization $\left(30 \mathrm{~min}\right.$ at $\left.37^{\circ} \mathrm{C}\right)$, highly hydrated hydrogels, with a fibrillar collagen density of less that $0.5 \% \mathrm{w} / \mathrm{v}$, were placed on a stack of blotting paper, nylon, and stainless steel meshes. Dense collagen gel scaffolds, with fibrillar collagen density of more than $10 \% \mathrm{w} / \mathrm{v}^{13}$, were produced by the application of an unconfined compressive stress of $1 \mathrm{kPa}$ for $5 \mathrm{~min}$ to remove excess casting fluid. The compressed scaffolds were circularly cut ( $5 \mathrm{~mm}$ diameter) and kept up to $24 \mathrm{~h}$ at $37^{\circ} \mathrm{C}$ under $5 \% \mathrm{CO}_{2}$ in serum-free medium before implantation. 
Surgical implantation, experimental procedure and sampling. Wistar male rats (12 week-old, $\sim 300 \mathrm{~g}$ ) were anesthetized $(100 \mathrm{mg} / \mathrm{kg}$ b.w. of ketamine and $10 \mathrm{mg} / \mathrm{kg}$ b.w. of xylazine hydrochloride, both from Centravet Alfort, Maisons-Alfort, France). In each specimen, scalp skin was incised, and the periosteum was eliminated to visualize the skull. A 5-mm-diameter calvarial critical-sized defect was created on each side of the parietal bone using a dental bur attached to a slow-speed hand piece operating at $1500 \mathrm{rpm}$, under irrigation with sterile saline solution ${ }^{36}$. Special care was taken for the sagittal suture preservation, and minimal invasion of the dura mater. After gently removing the circular bone plug, a rDPSC-seeded dense gel scaffold $(\mathrm{n}=15)$ was placed on one side of the rat skull and an acellular dense scaffold $(n=15)$ was placed on the opposite side, each rat being its own control. Defects created in the skulls of two additional rats were left empty as negative controls for the critical-size defect. Wound closure was achieved by a two layer suturing (periosteum, skin) using absorbable sutures (Vicryl Rapid 5.0 and 4.0 respectively, Ethicon, Johnson \& Johnson). Immediate post-operative care included analgesia with buprenorphine $(0.02 \mathrm{mg} / \mathrm{kg} \mathrm{b.w.)}$. After surgery, the animals were housed individually under constant conditions. No lethality was detected during the surgery or the post-operative period. Wound healing progressed without any sign of infection, material exposure or other complication. Body weights were examined regularly to ensure proper feeding before and after surgery.

At days 7, 14,21, 28 and 35 post surgery, animals were imaged using a micro-CT as described below. Three animals were euthanized at each time point, the calvarium was excised and separated into hemicalvarium. Samples were fixed in $70 \% \mathrm{v} / \mathrm{v}$ ethanol $\left(24\right.$ hours at $\left.4{ }^{\circ} \mathrm{C}\right)$, dehydrated in graded ethanol solutions, and embedded at $-20^{\circ} \mathrm{C}$ in methyl methacrylate resin (Merck) without decalcification ${ }^{37}$. Resin embedded hemicalvarium bone samples were cut ( $5 \mu \mathrm{m}$ thick) using a Jung Polycut E microtome (Leica) with hard tissue blades (Leica). After immersion in a drop of $50 \% \mathrm{v} / \mathrm{v}$ ethanol, sections were stretched to a fold-free state on polysine glass slides (Menzel-Gläser), covered with a polyethylene sheet, and tightly pressed on the glass slides, followed by overnight drying at RT. Deplastification was carried out in 2-methoxyethyl acetate (Carlo Erba) three times for 20 minutes. Rehydration of the sections was performed in graded ethanol solutions for subsequent procedures.

Micro-X-ray computed tomography (Micro-CT) examination of samples. For bone regeneration exploration, rats were anesthetized (isoflurane, induction at $3-4 \%$ under airflow of $0.8-1.5 \mathrm{~L} / \mathrm{min} ; 1.5-2 \%$ under $400-800 \mathrm{ml} / \mathrm{min}$ thereafter). They were imaged using an X-ray micro-CT device (Quantum FX Caliper, Life Sciences, Perkin Elmer, Waltham, MA) hosted by the PIV Platform, EA2496, Montrouge, France. The X-ray source was set at $90 \mathrm{kV}$ and $160 \mu \mathrm{A}$. Tridimensional images were acquired with an isotropic voxel size of $20 \mu \mathrm{m}$. An internal density phantom, calibrated in mg of hydroxyapatite, was used to scale bone density. Full 3D high-resolution raw data are obtained by rotating both the X-ray source and the flat panel detector $360^{\circ}$ around the sample (scanning time: $3 \mathrm{~min}$ ). Tridimensional rendering were subsequently extracted from Dicom data frames using the open-source OsiriX imaging software (v5.7.1, distributed under LGPL license, Dr A. Rosset, Geneva, Switzerland). Quantification of the regenerated bone inside each defect was done using CTscan Analyzer software (Skyscan, release 1.13.5.1, Kontich, Belgium). A global volume of interest (VOI) was drowning by interpolating 2D region of interests on consecutive sections to isolate the defect area. The obtained interpolated VOI comprised only the remodeled bone defect area. A global thresholding was determined interactively for bone selection and to eliminate background noise. The following parameters were analyzed: bone density (in mg of hydroxyapatite), bone volume fraction BV/TV (\%), trabecular thickness Tb.Th $(\mathrm{mm})$, trabecular number Tb.N $(1 / \mathrm{mm})$, trabecular separation Tb.Sp $(\mathrm{mm})$ and trabecular pattern factor Tb.Pf $(1 / \mathrm{mm})^{38}$.

Histology examination of samples and histomorphometry. Deplastified hemicalvarium bone sample sections, ( $5 \mu \mathrm{m}$ thick) were sequentially cleared in water and stained with toluidine blue (pH 3.8), Von Kossa staining, Sirius red, or processed for alkaline phosphatase (ALP) enzymohistochemistry and for tartrate-resistant acid phosphatase (TRAP) revelation. Toluidine blue staining was used to visualize connective bone matrix. Von Kossa staining was used to visualize mineralized bone. Sirius red staining was used to visualize birefringent Type I collagen fibers in the bone marrow area. TRAP was detected by using hexazotized pararosanilin (Sigma) and naphtol ASTR phosphate (Sigma, St Louis, MO) to reveal osteoclasts; non-osteoclastic acid phosphatase was inhibited by adding $100 \mathrm{mMol} / \mathrm{L} \mathrm{L}(+)$-tartric acid (Sigma, St Louis, MO) to the substrate solution. Image acquisition was performed using a DMLB Leica microscope, equipped with imaging camera DFC425 Leica connected to the Leica application (LAS version 4.4) and image analysis was performed using ImageJ.

In situ hybridization analysis. RNA extractions were performed using RNeasy Plus mini kit (Qiagen, Venlo, Netherlands), and cDNA were synthesized using the Verso cDNA synthesis kit (ThermoFicher, Waltham, MA). RNA probes were designed using SP6 and T7-tailed oligonucleotides for Colla1 (forward primer 5'ATTTAGGTGACACTATAGGAACAAGGTGACAGAGGCAT 3' and reverse primer 5' TAATACGACTCACTATAGGGTTGGTTAGGGTCGATCCAGT 3'), for a total length amplified between primers of $540 \mathrm{bp}$. Sense and antisens RNA probes were synthesized and labeled with Digoxigenin-UTP (Roche, Inc.) using the Riboprobe ${ }^{\circledR}$ Combination System -SP6/T7 RNA Polymerase kit (Promega) following the manufacturer's instructions. The mixture contained the following nucleotides: $0.1 \mathrm{mM}$ of rATP, $0.1 \mathrm{mM}$ of rCTP, $0.1 \mathrm{mM}$ of rGTP, $0.05 \mathrm{mM}$ of rUTP, $0.05 \mathrm{mM}$ of digoxigenin-11-dUTP (Roche), and $800 \mathrm{ng}$ of rat cDNA as templates. Probes were purified using the kit Illustrated ProbeQuant G-50 Micro Columns (GE Healthcare).

Deplastified and PBS 1X rehydrated hemi-calvarium bone sample sections ( $5 \mu \mathrm{m}$ thick) were permeabilized with $10 \mu \mathrm{g} / \mathrm{ml}$ proteinase $\mathrm{K}$ (Sigma) in $\mathrm{PBS}$ at $37^{\circ} \mathrm{C}$ for 20 minutes, and post-fixed in $4 \%$ neutral-buffered formalin for 30 minutes at room temperature. After washing in PBS for 5 minutes, followed by washes SCC 2X, hybridization was performed in a humid chamber at $65^{\circ} \mathrm{C}$ overnight in a mixture containing the labeled RNA probe at $1 / 100$ in $1 \mathrm{mg} / \mathrm{ml}$ Yeast ARNt, $50 \%$ formamide, $10 \%$ dextran sulfate, SCC $5 \mathrm{X}$ and Denhardt's $1 \mathrm{X}$. After hybridization, excess probe was eliminated by washing the sections for 30 minutes in buffer with 50\% formamide in SCC 1X and 

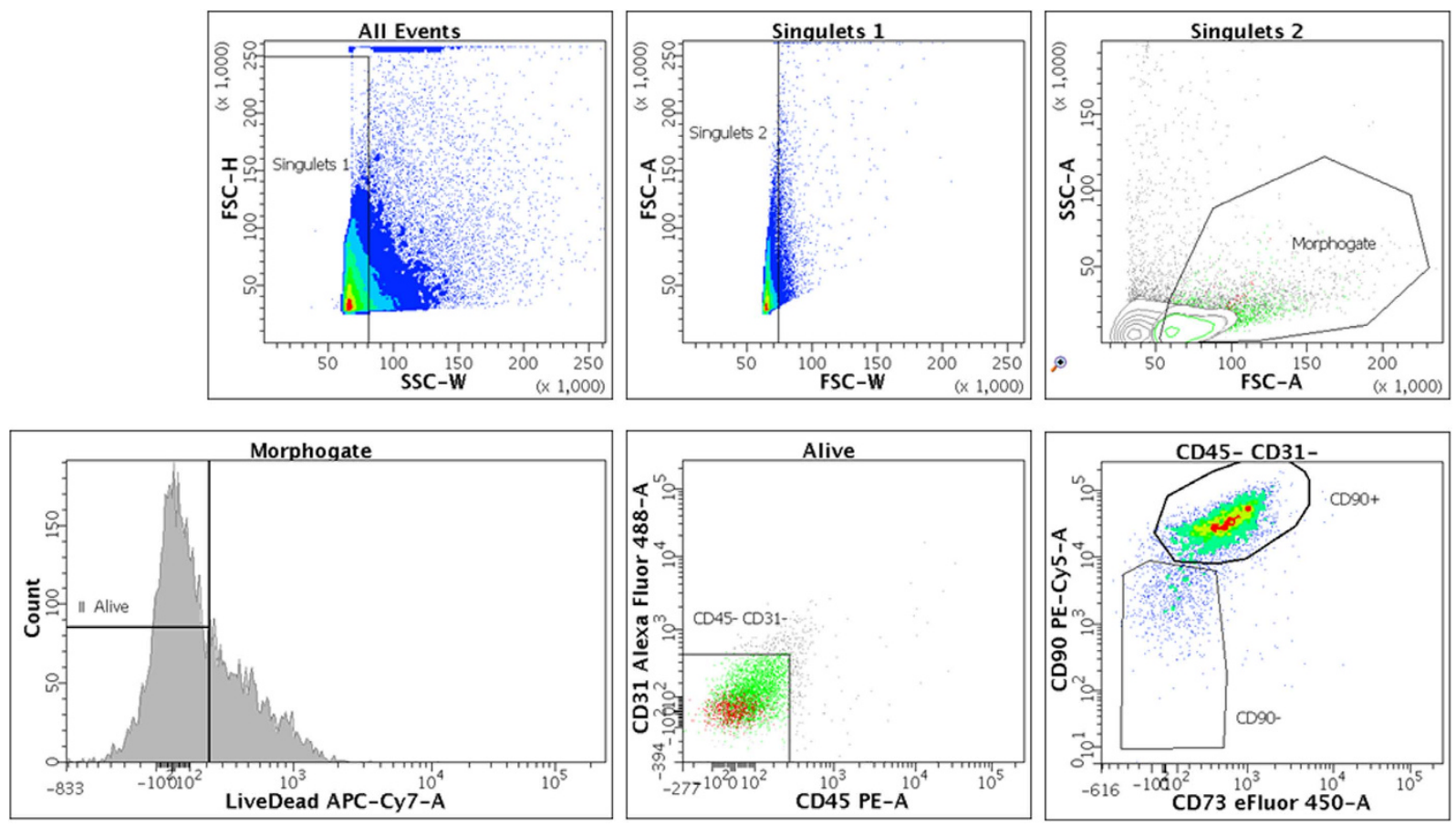

Figure 1. rDPSCs phenotype by flow cytometry. The expression of a series of cell surface markers associated with the mesenchymal stem cell (MSC) phenotype was investigated using flow cytometry. Passage 2 rDPSCs, represented by events from alive cells in both morphogate and singulet gates, were negative for CD31 and CD45, and positive for CD73 and CD90, as expected for mesenchymal stromal cells.

$0.1 \%$ Tween 20 at $65^{\circ} \mathrm{C}$ for $30 \mathrm{~min}$, then twice for 1 hour. Then, tissue sections were equilibrated in a bath of MABT ( $100 \mathrm{mM}$ maleic acid, $0.5 \mathrm{M} \mathrm{NaCl}, 0.1 \%$ Tween 20 ) at room temperature for 30 minutes. After 2 hours in blocking solution (20\% inactivated goat serum, 2\% Blocking Reagent [Roche] in MABT), the presence of Colla1 in tissue sections was researched by immunoreactivity with an anti-digoxigenin-AP, Fab fragments (Roche Diagnostic) at 1/1000 in blocking solution, overnight at room temperature. After rinsing in MABT five times for $30 \mathrm{~min}$ utes, the presence of Colla1 in tissue sections was revealed using NBT $(0.45 \mu \mathrm{l} / \mathrm{ml}$, Roche $)$ and BCIP $(3.5 \mu \mathrm{l} / \mathrm{ml}$, Roche). The tissue sections were then rinsed and covered with a coverslip mounted Gel Mounting Medium (Dako Cytomation). Image acquisition was performed using a DMLB Leica microscope, equipped with imaging camera DFC425 Leica connected to the Leica application (LAS version 4.4).

Statistical analysis. Results in each group were expressed as the mean \pm standard error of the mean (S.E.M.). Variables were compared using one-way analysis of variance (ANOVA) followed with two-by-two comparisons performed with paired T-test of Student when they passed the Fisher F equal variance and Shapiro-Wilk normality tests. Otherwise, they were performed using Kruskal-Wallis ANOVA on the ranks followed with two-by-two comparisons performed with the U test of Mann and Whitney. Statistical significance was set at $\mathrm{p}<0.05$.

\section{Results}

rDPSCs displayed a mesenchymal stromal cell phenotype. The expression of a series of cell surface markers associated with the mesenchymal stem cell (MSC) phenotype was investigated using flow cytometry. Most of passage 2 rDPSCs, represented by events from alive cells in both morphogate and singulet gates, were negative for CD31 and CD45, and >75\% were positive for CD73 and CD90, as expected for mesenchymal stromal cells (Fig. 1) $)^{39,40}$.

Dense collagen gel scaffolds seeded with rDPSCs improved bone healing process under Micro-X-ray computed tomography. To evaluate bone regeneration, the parietal bone was examined by micro-CT scan. Thirty-five days after surgery, control empty defects showed a thin non-mineralized healing connective tissue with no sign of bone regeneration. Parietal defects filled with either rDPSC-seeded or acellular dense gel scaffolds demonstrated a progressive amount of new bone formation starting from the edges as well as from the center of the defect. No inflammation was observed in the seeded scaffold implants. Micro-CT analysis of 3D micro-architecture parameters of the remodeled bone defect area are summarized in Fig. 2B. Compared to acellular scaffolds, denser and better-organized trabeculae were observed in rDPSC-seeded scaffolds whereas none of the defects showed complete closure at day 35 (Fig. 2A). Scaffolds seeded with rDSPCs demonstrated a significantly higher amount of calcified material within the remodeled defects when compared to acellular scaffolds from day 21 post surgery (density ratio of $2.5 \pm 1.3 v s .0 .9 \pm 0.2$ at day $21, \mathrm{p}<0.05$; and $4.2 \pm 1.5$ vs. $0.8 \pm 0.2$ at day $35, \mathrm{p}<0.01$; respectively). Scaffolds with rDPSCs led to significantly higher mineralized tissue volume BV/ 
A

Empty defects
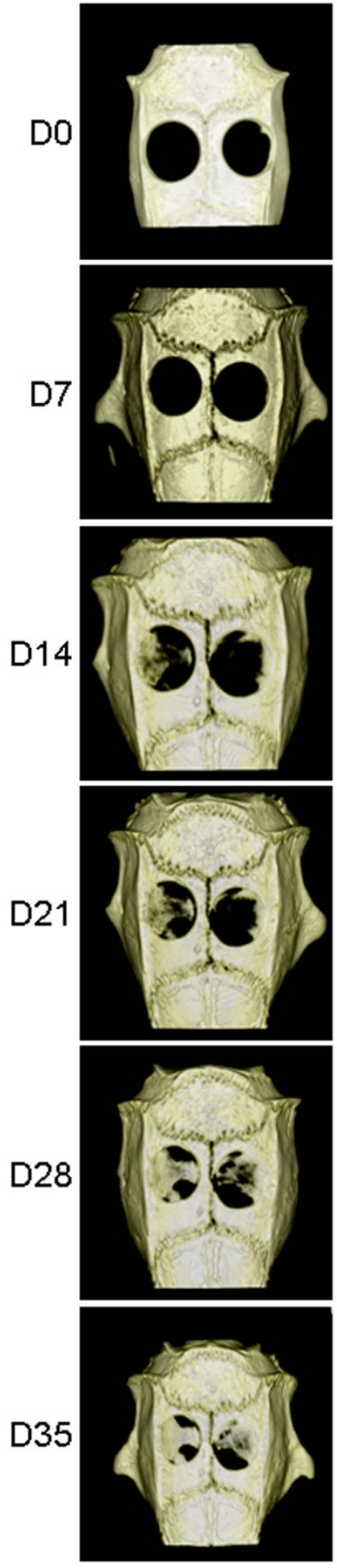

Scaffold-filled defects
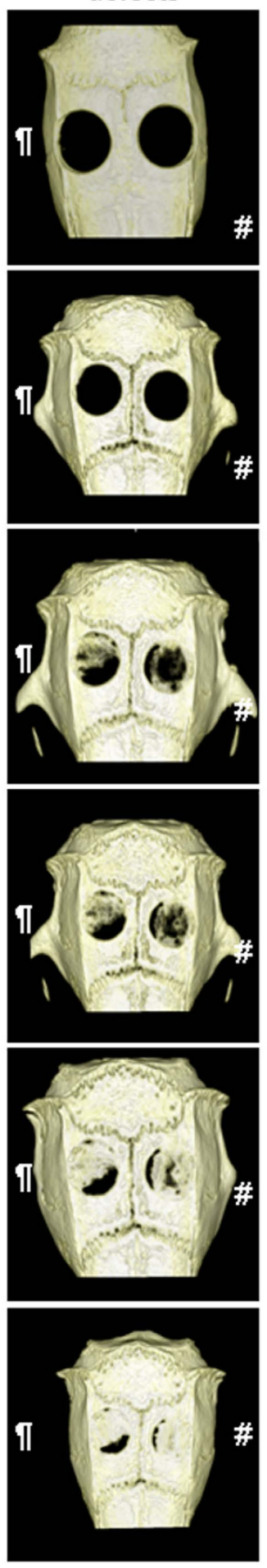

B
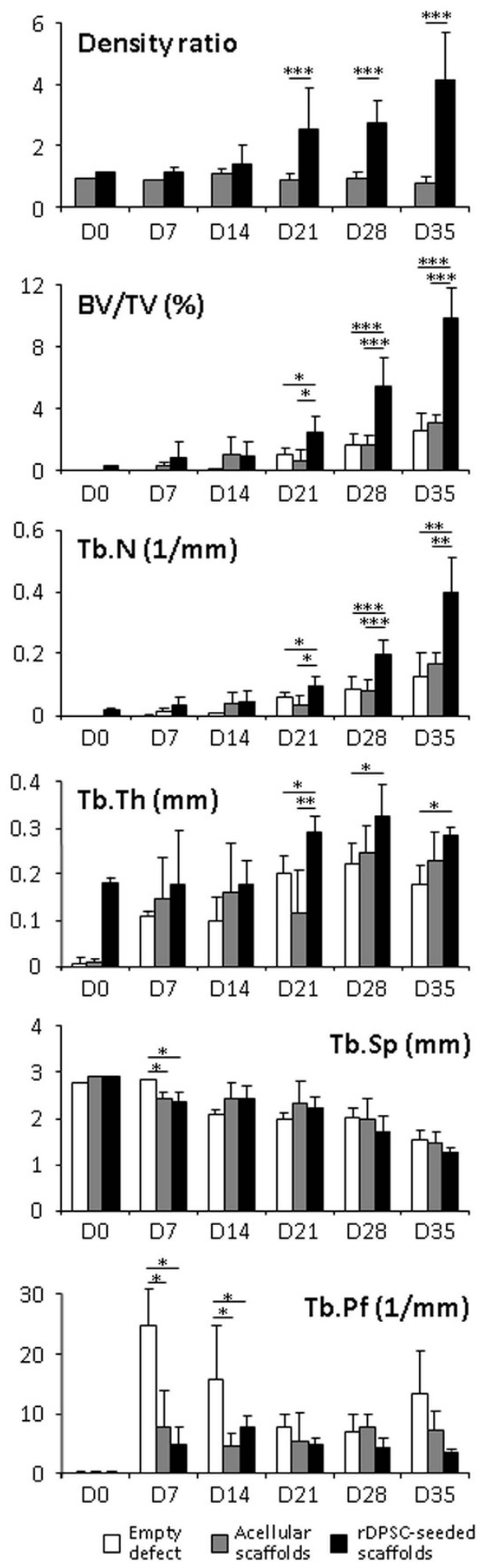

Figure 2. Micro-CT analysis (A) Rat skull 3D rendering at D0 to D35. Calvarial defects that were left empty did not heal spontaneously for the duration of the study. In contrast, bone healing was gradually achieved when the calvarial defects were filled with either rDPSC-seeded (\#) or acellular dense collagen gel scaffolds ( $(\mathbf{g})$.(B) Bone density and micro-architectural parameters. Bone volumetric fractions were expressed as a percentage of bone volume on the total area of the defect. Bone parameters were quantified as a $\%$ of tissue volume (BV/TV) within the defected area, trabecular number (Tb.N), trabecular thickness (Tb.Th), trabecular separation (Tb.Sp) and trabecular pattern factor (Tb.Pf). ${ }^{*} \mathrm{p}<0.05 ;^{* *} \mathrm{p}<0.01{ }^{* * *} \mathrm{p}<0.001$. 


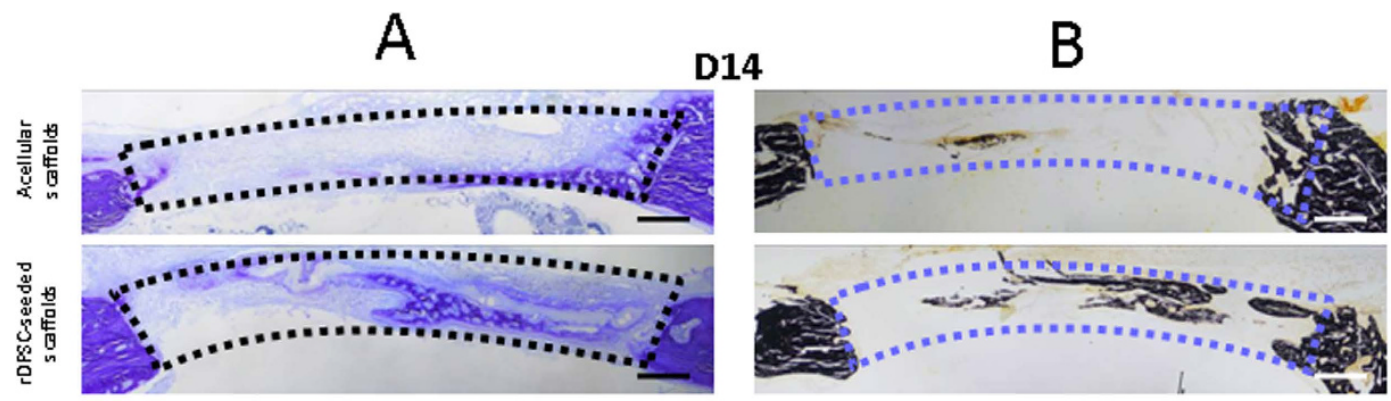

D21
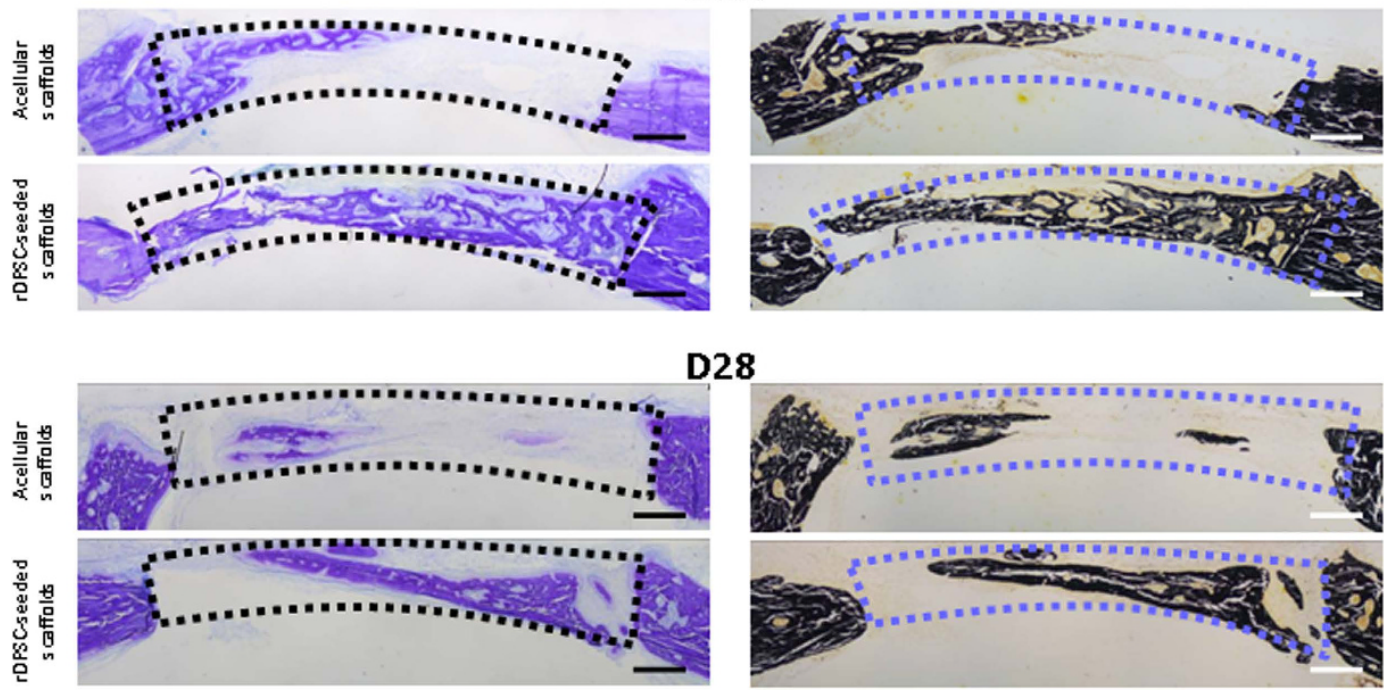

D28

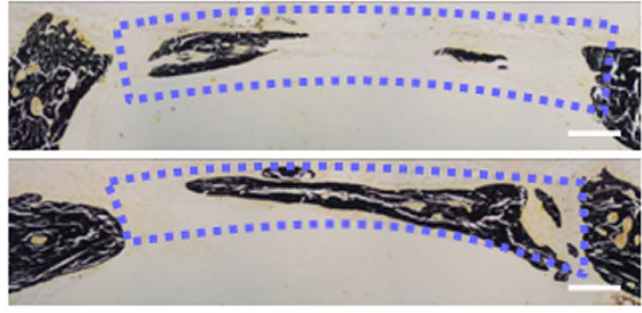

D35
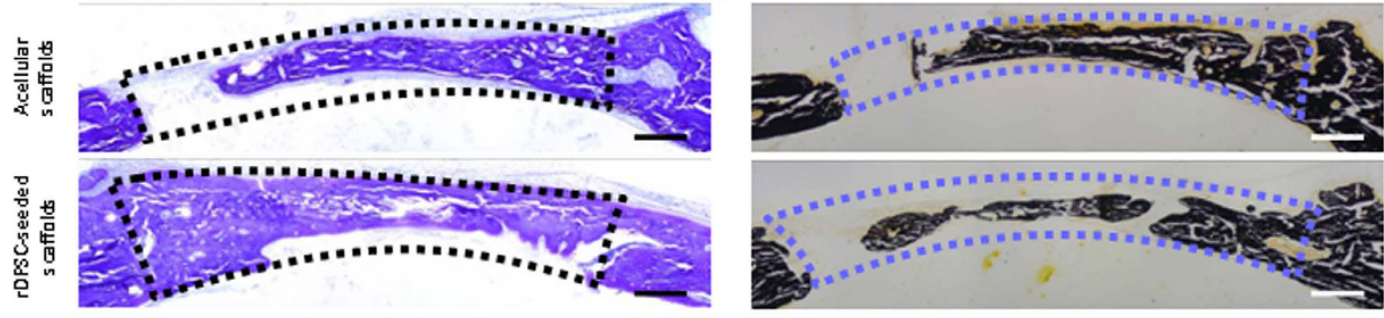

Toluidin blue (\%)
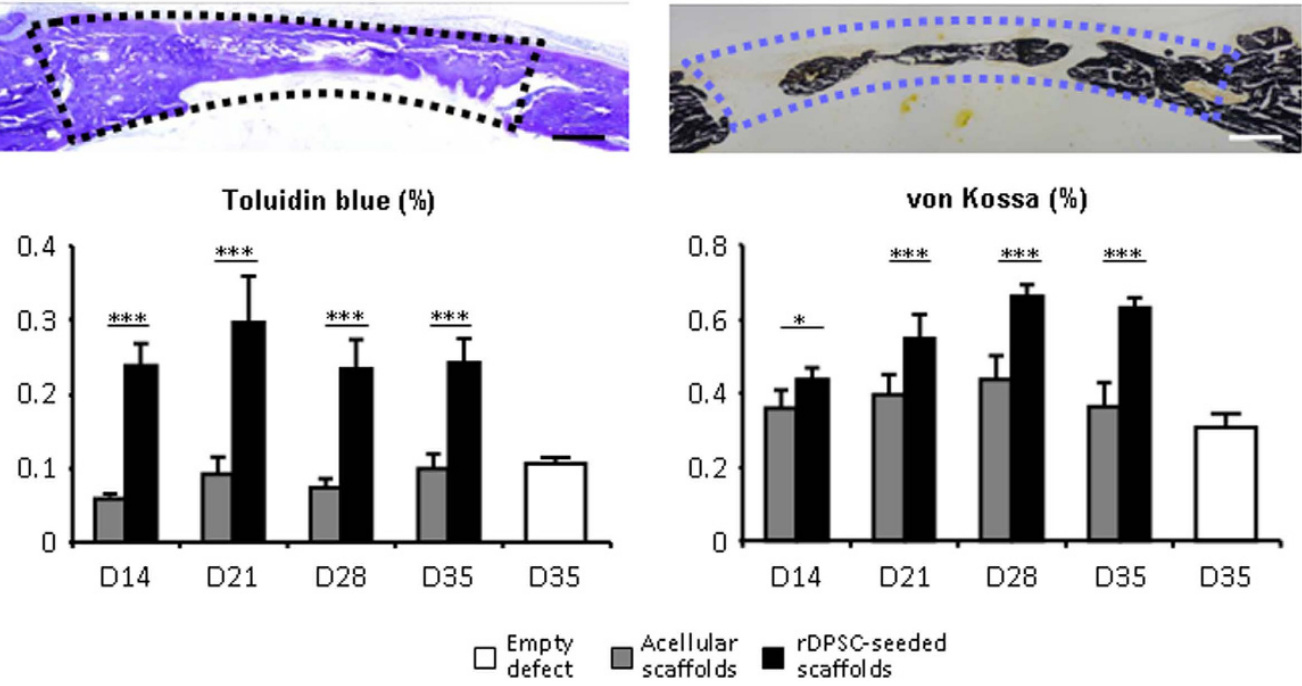

Figure 3. Histology and histomorphometry. Representative and resin embedded calcified bone stained with Toluidine Blue (A) and von Kossa (B) images are shown at days 14 to 35 post-operative for defects filled with rDPSC-seeded dense collagen gel scaffolds $(n=15)$ and acellular scaffolds $(n=15)$. Compared to acellular scafoolds, there was a significant increase in the percentage area (underlined by dotted lines) of Toluidine Blue and von Kossa positive staining in defects filled with rDPSC-seeded dense collagen gel scaffolds. No significant difference was observed at day 7 (data not shown). Scale bars represent $500 \mu \mathrm{m} .{ }^{\star} \mathrm{p}<0.05 ;{ }^{* *} \mathrm{p}<0.001$. 
A

A Acellular scaffolds

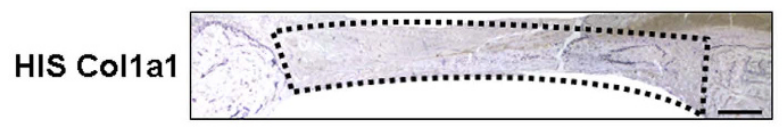

SR
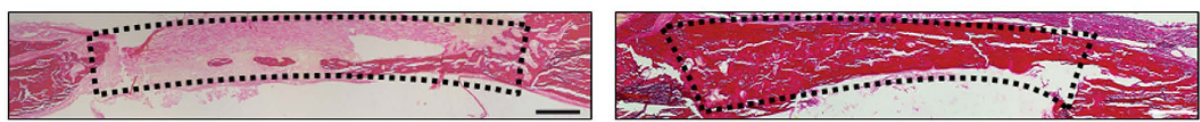

SR Pol
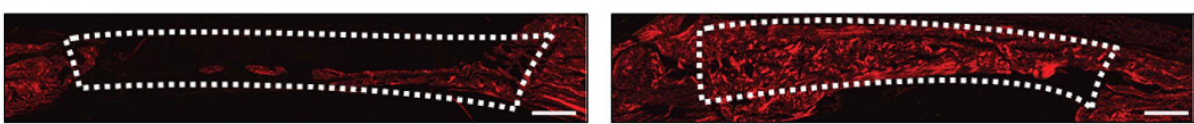

B

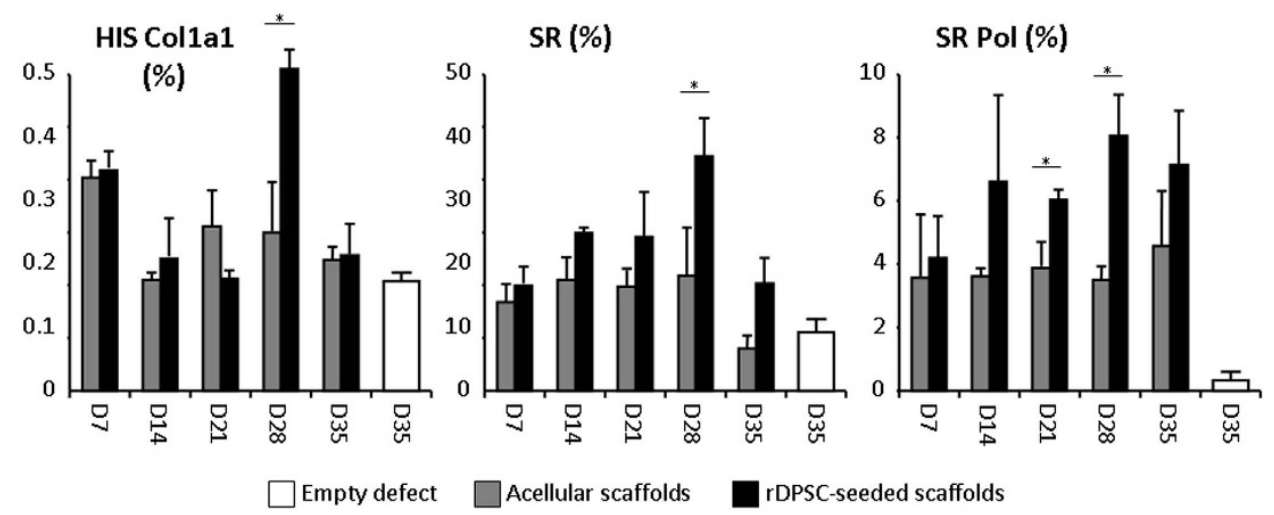

Figure 4. In situ hybridization and histology analysis of the collagen component during bone regeneration. In situ hybridization (HIS) studies using the Collal probe reacting with recipient osteoblasts and osteocytes associated within the new bone formation showed strong collagen synthesis in defects filled with rDPSC-seeded dense collagen gel scaffolds, whereas only weak Colla1-associated signals were observed in defects filled with acellular scaffolds. Sirius red (SR) stainings were performed to visualize the collagen protein neo-secretion when using polarized light $(\mathrm{Pol})$. Representative images at day 28 post-operative $(\mathbf{A})$ and quantification from days 14 to 35 (B) are shown for defects filled with rDPSC-seeded dense collagen gel scaffolds $(n=15)$ and acellular scaffolds $(n=15)$. Scale bars represent $500 \mu \mathrm{m}$. Dotted lines represent the analyzed areas. No significant difference was observed at day 7 (data not shown). ${ }^{*} \mathrm{p}<0.05$.

TV and trabecular number Tb.N than acellular scaffolds or empty defects from day 21, without affecting the trabecular thickness Tb.Th, separation Tb.Sp and trabecular pattern factor Tb.Pf. In other words, the defected area acquired a higher mineralized healing tissue with higher trabecular structure when rDPSC-seeded scaffolds were used, suggesting a stimulatory effect of pulp stem cells on calvaria healing.

Restored bone remodeling processes in scaffold-filled defects. To investigate the remodeled tissue within the bone defect area, fibrous connective tissue and mineralized deposits were stained with toluidine blue and von Kossa, respectively. New connective and bone tissue formation were calculated as a percentage of filling from the baseline defect. Histological evidence further supported the micro-CT findings, indicating that rDPSC-seeded scaffolds demonstrated a progressive presence of fibrous and mineralized connective tissue within the remodeled defect. The newly formed bone had a typical organized and mature bone morphology with noticeable marrow spaces and similar to native bone (Fig. 3). In contrast, histological analysis indicated a small amount of irregularly arranged bone tissue and less bone formation in acellular scaffolds. The percentage of new connective bone tissue visualized with toluidine blue area was significantly higher $(\mathrm{p}<0.001)$ at days 14, 21, 28 and 35 post surgery when using rDPSC-seeded scaffolds, compared to both acellular scaffolds and empty defects. Similarly, the percentage of new mineralized tissue bone area evidenced with von Kossa was significantly higher at days $14(\mathrm{p}<0.05)$ and 21,28 and 35 post surgery $(\mathrm{p}<0.001)$, when using rDPSC-seeded scaffolds, compared to both acellular scaffolds and empty defects.

In situ hybridization studies using the specific Collal probe indicated strong collagen expression in rDPSC-seeded scaffolds, especially at the exterior and interior periosteal sides of the newly formed calvarium diploe, whereas only weak Colla1-associated signals were observed in acellular scaffolds (Fig. 4). This increased Collal gene expression was further supported with the comparison of Sirius red staining that marks the collagen fibers. This staining was much stronger in rDPSC-seeded scaffolds when compared with the acellular group, indicating that more collagen protein was secreted in the rDPSC group. In addition, the investigation of ALP and TRAP activities revealed a restored osteoblastic forming and osteoclastic resorbing processes within new mineralized tissue bone area, especially when rDPSC-seeded scaffolds were implanted (Fig. 5). 
Acellular scaffolds

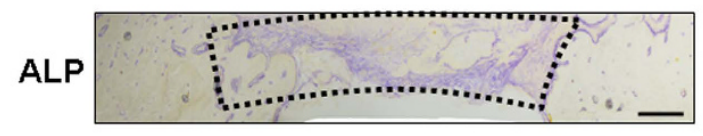

TRAP

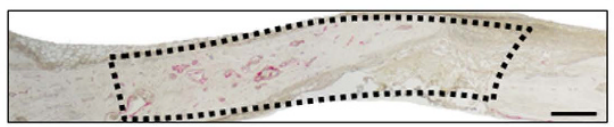

\section{rDPSC-seeded scaffolds}
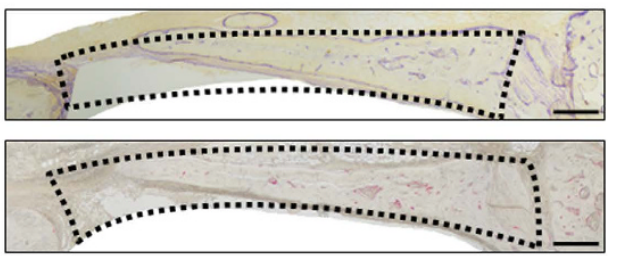

B

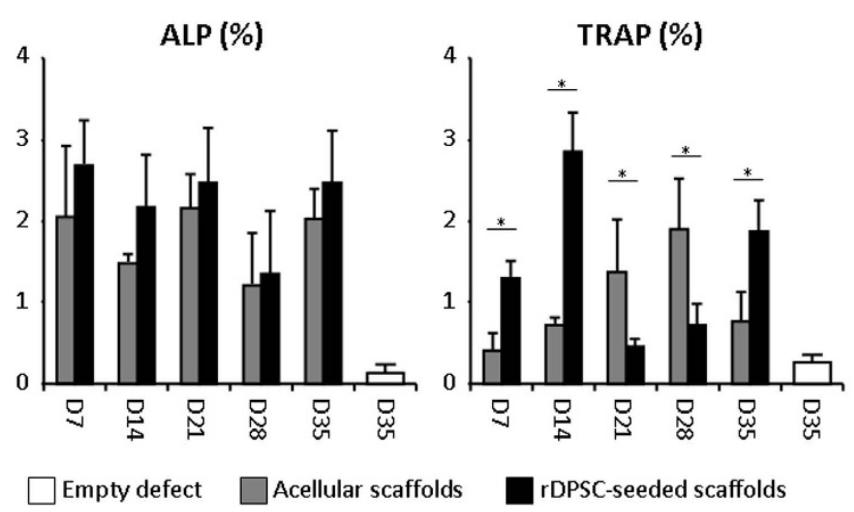

Figure 5. Immunohistochemical analysis of bone remodeling component during bone regeneration. Staining of osteoblastic-associated alkaline phosphatase (ALP) and osteoclastic-associated tartrate-resistant acid phosphatase (TRAP) activities revealed osteoblastic forming and osteoclastic resorbing processes within new mineralized bone tissue area, in particular when rDPSC-seeded scaffolds were implanted. Representative images at day 28 post-operative (A) and quantification from days 14 to 35 (B) are shown for defects filled with rDPSC-seeded dense collagen gel scaffolds $(n=15)$ and acellular scaffolds $(n=15)$. Scale bars represent $500 \mu \mathrm{m}$. Dotted lines represent the analyzed areas. No significant difference was observed at day 7 (data not shown). ${ }^{*} \mathrm{p}<0.05$.

\section{Discussion}

Craniofacial area is particularly vulnerable to defects or bone loss because of trauma, pathologies or hereditary malformation, for which bone reconstruction is particularly difficult. Here, we show that dense collagen scaffolds combined with mesenchymal stem cells derived from the dental pulp drastically enhanced bone regeneration in a calvarial defect model, suggesting that this association represents an effective therapeutic tool to regenerate craniofacial bones.

In this study, a dense collagen gel scaffold was used as a carrier for $\mathrm{CD} 31^{-} \mathrm{CD} 45^{-} \mathrm{CD} 73^{+} \mathrm{CD} 90^{+}$rDPSCs to assess their bone regeneration effects on the very thin rat skull. This naturally-derived biomimetic biomaterial, that resembles the organic or "osteoid" phase of native bone, was simply generated through rapid removal of fluid from highly-hydrated collagen gels using plastic compression ${ }^{13}$, where cell seeding is part of the scaffolds processing. This natural scaffold has been reported to exhibit osteoinductive and osteoconductive abilities ${ }^{14,15,34,41,42}$ that are critical for osteointegration into the bone, especially for craniomaxillofacial applications. Through the increased collagen fibrillar density, a native matrix scaffold that mimics the microstructure (and stiffness) of the osteoid is produced, which provides both biological and mechanical cues to accelerate osteogenic differentiation of three-dimensionally seeded cells. Furthermore, the clinical use of collagen scaffolds is successful and widely accepted with excellent biodegradation, and biocompatibility ${ }^{43-45}$. In particular, previous studies have shown that collagen-based scaffolds are substitutable materials ${ }^{46}$, which in combination with MSCs can promote mineralization and heal a large bone unicortical defect ${ }^{16}$. In this study, authors used osteogenic pre-conditioned mesodermal bone marrow derived MSC, seeded into a compressed type I collagen scaffold, to heal a mesodermal appendicular weight bearing bone (femur) after an unicortical defect in the mouse. Furthermore, VEGF was injected into the femoral canal at the level of the defect to enhance angiogenesis ${ }^{16}$. Here, both the mesenchymal cell embryologic origin and the damaged bone specificity differed from this previous study. Indeed, we evaluated for the first time the osteogenic effects of the association of a dense collagen gel scaffolds seeded with non-pre-conditioned neural crests derived DPSCs on bone regeneration in a rat critical-size calvarial defect model, a non-weight-bearing bone formed by intramembranous ossification. In addition, no VEGF was used to enhance angiogenesis.

Critical-sized bone defect are currently the reference models in exploring the efficiency of osteoconductive substitutes on healing and regeneration ${ }^{36}$. The bilateral rat calvarial defect model used in this study is highly reproducible. Two critical-sized defects were created on either side of the parietal region and filled with either rDPSC-seeded, or acellular dense collagen gel scaffold, whereby each rat was its own control. At day 35 post surgery, rDPSC-seeded dense collagen gel scaffold displayed osteogenic effects enhancing bone healing, which was in contrast to the control group that did not show bone regeneration, confirming that the selected defect model was critical over the duration of the study. 
Contrary to a previous report ${ }^{31}$, graft rejection was not observed in the present study. Stem cells from dental sources are interesting with the ease of accessibility, and high proliferation/differentiation capacities ${ }^{47}$. Indeed, osteogenic differentiation of DPSCs seeded in dense collagen gel scaffolds was another important factor in the potential success of bone regeneration. Since their first isolation in $2000^{24}$, DPSCs deriving from neural crests ${ }^{22}$ have been reported to have a higher proliferation rate than bone marrow stem cells ${ }^{5}$ and adipose-derived stem cells $^{48,49}$. Similar to mesenchymal stromal cells, DPSCs are reported to be positive for CD73 and CD90 (two mesenchymal stem cell markers), and negative for CD31 (a marker of endothelial lineage) and CD45 (a marker of hematopoietic lineage $)^{21,22}$. Furthermore, autologous DPSCs have been shown to promote bone regeneration when used in association with a collagen sponge ${ }^{6}$. Since wisdom teeth extraction is one of the most common procedures in oral surgery, the harvested DPSCs can be per se considered as a powerful source of stem cells useful for autologous tissue engineering ${ }^{50}$ with a positive impact on bone regeneration ${ }^{32,51,52}$. Pathologic features of the craniomaxillofacial skeleton, also mainly a neural crest-derived tissue, could possibly be corrected with stem cell therapies. Owing to their similar embryonic origins, the potential of DPSCs as an autologous stem cell source for treating skull defects was investigated.

The samples in this study were investigated both by histology and by micro-computed tomography. Micro-CT analysis has become a major technique of investigation in the assessment of bone regeneration/healing in bone defect models, with several advantages such as rapid calculation and multiple analysis opportunities ${ }^{20,53}$. Histomorphometric examinations are reliable and widely used in the assessment of bone tissue regeneration/ healing. In the current study, the dense collagen-based scaffold supported the long-term metabolic activity of DPSCs to promote the recruitment and differentiation of osteoblasts that deposited a calcium-phosphate rich mineral in the extracellular matrix, associated with the expression of type I collagen. The presence of a fibrous and mineralized scaffold, stained with toluidine blue and von Kossa, localized in the centre of the defect indicated that osteoinduction was, at least in part, initiated or promoted by cells trapped in the scaffold. This was also further supported by the strong osteoblast-associated ALP staining within and at the periphery of the healing defect. Together with the presence of TRAP-positive osteoclasts in and around the scaffold, this suggests an early active and restored matrix turnover within the initial defect, especially when rDPSC-seeded dense scaffolds were used. The osteoconductive properties of the mineralized scaffolds, aided by DPSCs, would thus enable migration and attachment of endogenous bone forming progenitor cells. The presence of both osteoblasts and osteoclasts in central and peripheral areas further suggests the extracellular matrix within the bone defect was undergoing active turnover. Additional studies aimed at determining the fate of transplanted DPSCs and their relationship with endogenous cells during defect repair will require the use of a tracking system or other labeled cells in the scaffold. A detailed time course, with long-term bone healing effects (i.e. $>5$ weeks) will be necessary to reveal the molecular mechanisms underlying the increase in bone formation observed in the current study at one month.

\section{Conclusions}

This study has demonstrated the potential of neural crest derived DPSC-loaded-dense collagen gel scaffolds to benefit the craniofacial bone healing process. The association of DPSCs with appropriate physiological osteogenic scaffold (dense collagen gel) is a promising approach when using tissue-engineering techniques for clinically relevant large craniofacial bone defects and opens new avenues for further research aiming to improve cell cultivation and scaffold design.

\section{References}

1. Riccio, M. et al. Fibroin scaffold repairs critical-size bone defects in vivo supported by human amniotic fluid and dental pulp stem cells. Tissue Eng Part A 18, 1006-1013 (2012).

2. Barone, A. \& Covani, U. Maxillary alveolar ridge reconstruction with nonvascularized autogenous block bone: clinical results. J Oral Maxillofac Surg 65, 2039-2046 (2007).

3. Nkenke, E. \& Neukam, F. W. Autogenous bone harvesting and grafting in advanced jaw resorption: morbidity, resorption and implant survival. Eur J Oral Implantol 7 Suppl 2, S203-217 (2014).

4. Zhong, W. et al. In vivo comparison of the bone regeneration capability of human bone marrow concentrates vs. platelet-rich plasma. PLoS One 7, e40833 (2012).

5. Delloye, C., Cornu, O., Druez, V. \& Barbier, O. Bone allografts: What they can offer and what they cannot. J Bone Joint Surg Br 89, 574-579 (2007)

6. Bauer, T. W. \& Muschler, G. F. Bone graft materials. An overview of the basic science. Clin Orthop Relat Res 10-27 (2000).

7. Saha, S. et al. Osteochondral tissue engineering in vivo: a comparative study using layered silk fibroin scaffolds from mulberry and nonmulberry silkworms. PLoS One 8, e80004 (2013).

8. Helary, C. et al. Concentrated collagen hydrogels as dermal substitutes. Biomaterials 31, 481-490 (2010).

9. Helary, C. et al. Evaluation of dense collagen matrices as medicated wound dressing for the treatment of cutaneous chronic wounds. Biomaterials science 3, 373-382 (2015).

10. Abou Neel, E. A. et al. Collagen-emerging collagen based therapies hit the patient. Adv Drug Deliv Rev 65, 429-456 (2013).

11. Mravic, M., Peault, B. \& James, A. W. Current trends in bone tissue engineering. Biomed Res Int 2014, 865270 (2014).

12. Cheema, U. et al. Fabricating tissues: Analysis of farming versus engineering strategies. Biotechnology and Bioprocess Engineering 12, 9-14 (2007).

13. Brown, R. A., Wiseman, M., Chuo, C. B., Cheema, U. \& Nazhat, S. N. Ultrarapid Engineering of Biomimetic Materials and Tissues: Fabrication of Nano- and Microstructures by Plastic Compression. Advanced Functional Materials 15, 1762-1770 (2005).

14. Bitar, M., Salih, V., Brown, R. A. \& Nazhat, S. N. Effect of multiple unconfined compression on cellular dense collagen scaffolds for bone tissue engineering. J Mater Sci Mater Med 18, 237-244 (2007).

15. Buxton, P. G. et al. Dense collagen matrix accelerates osteogenic differentiation and rescues the apoptotic response to MMP inhibition. Bone 43, 377-385 (2008).

16. Gao, C. et al. MSC-seeded dense collagen scaffolds with a bolus dose of VEGF promote healing of large bone defects. Eur Cell Mater 26, 195-207, discussion 207 (2013).

17. Chicatun, F. et al. Osteoid-mimicking dense collagen/chitosan hybrid gels. Biomacromolecules 12, 2946-2956 (2011).

18. Engler, A. J., Sen, S., Sweeney, H. L. \& Discher, D. E. Matrix elasticity directs stem cell lineage specification. Cell 126, 677-689 (2006). 
19. Ghezzi, C. E., Muja, N., Marelli, B. \& Nazhat, S. N. Real time responses of fibroblasts to plastically compressed fibrillar collagen hydrogels. Biomaterials 32, 4761-4772 (2011).

20. Annibali, S. et al. Micro-CT and PET analysis of bone regeneration induced by biodegradable scaffolds as carriers for dental pulp stem cells in a rat model of calvarial "critical size" defect: Preliminary data. Journal of Biomedical Materials Research Part B: Applied Biomaterials 102, 815-825 (2014).

21. Abe, S., Hamada, K., Miura, M. \& Yamaguchi, S. Neural crest stem cell property of apical pulp cells derived from human developing tooth. Cell Biol Int 36, 927-936 (2012).

22. Chai, Y. et al. Fate of the mammalian cranial neural crest during tooth and mandibular morphogenesis. Development 127, 1671-1679 (2000).

23. d'Aquino, R. et al. Human postnatal dental pulp cells co-differentiate into osteoblasts and endotheliocytes: a pivotal synergy leading to adult bone tissue formation. Cell Death Differ 14, 1162-1171 (2007).

24. Gronthos, S., Mankani, M., Brahim, J., Robey, P. G. \& Shi, S. Postnatal human dental pulp stem cells (DPSCs) in vitro and in vivo. Proc Natl Acad Sci USA 97, 13625-13630 (2000).

25. Kerkis, I. \& Caplan, A. I. Stem cells in dental pulp of deciduous teeth. Tissue Eng Part B Rev 18, 129-138 (2012).

26. Tatullo, M., Marrelli, M. \& Paduano, F. The regenerative medicine in oral and maxillofacial surgery: the most important innovations in the clinical application of mesenchymal stem cells. Int J Med Sci 12, 72-77 (2015).

27. Zhao, H. \& Chai, Y. Stem Cells in Teeth and Craniofacial Bones. J Dent Res 94, 1495-1501 (2015).

28. d'Aquino, R. et al. Human mandible bone defect repair by the grafting of dental pulp stem/progenitor cells and collagen sponge biocomplexes. Eur Cell Mater 18, 75-83 (2009).

29. Hilkens, P., Meschi, N., Lambrechts, P., Bronckaers, A. \& Lambrichts, I. Dental Stem Cells in Pulp Regeneration: Near Future or Long Road Ahead? Stem Cells Dev 24, 1610-1622 (2015)

30. La Noce, M. et al. Dental pulp stem cells: state of the art and suggestions for a true translation of research into therapy. J Dent 42, 761-768 (2014).

31. De Miguel, M. P. et al. Immunosuppressive properties of mesenchymal stem cells: advances and applications. Curr Mol Med 12, 574-591 (2012).

32. de Mendonca Costa, A. et al. Reconstruction of large cranial defects in nonimmunosuppressed experimental design with human dental pulp stem cells. J Craniofac Surg 19, 204-210 (2008).

33. Gronthos, S., Arthur, A., Bartold, P. M. \& Shi, S. A method to isolate and culture expand human dental pulp stem cells. Methods Mol Biol 698, 107-121 (2011)

34. Coyac, B. R. et al. Mineralization of dense collagen hydrogel scaffolds by human pulp cells. J Dent Res 92, 648-654 (2013).

35. Rajan, N., Habermehl, J., Cote, M. F., Doillon, C. J. \& Mantovani, D. Preparation of ready-to-use, storable and reconstituted type I collagen from rat tail tendon for tissue engineering applications. Nat Protoc 1, 2753-2758 (2006).

36. Spicer, P. P. et al. Evaluation of bone regeneration using the rat critical size calvarial defect. Nat Protoc 7, 1918-1929 (2012).

37. Chappard, D., Palle, S., Alexandre, C., Vico, L. \& Riffat, G. Bone embedding in pure methyl methacrylate at low temperature preserves enzyme activities. Acta Histochem 81, 183-190 (1987).

38. Larsson, E. et al. In XIII Mediterranean Conference on Medical and Biological Engineering and Computing 2013 Vol. 41 IFMBE Proceedings (ed Roa Romero, Laura M.) Ch. 48, 194-197 (Springer International Publishing, 2014).

39. Lv, F. J., Tuan, R. S., Cheung, K. M. \& Leung, V. Y. Concise review: the surface markers and identity of human mesenchymal stem cells. Stem Cells 32, 1408-1419 (2014).

40. Hilkens, P. et al. Effect of isolation methodology on stem cell properties and multilineage differentiation potential of human dental pulp stem cells. Cell Tissue Res 353, 65-78 (2013).

41. Pedraza, C. E., Marelli, B., Chicatun, F., McKee, M. D. \& Nazhat, S. N. An in vitro assessment of a cell-containing collagenous extracellular matrix-like scaffold for bone tissue engineering. Tissue Eng Part A 16, 781-793 (2010).

42. Serpooshan, V. et al. Reduced hydraulic permeability of three-dimensional collagen scaffolds attenuates gel contraction and promotes the growth and differentiation of mesenchymal stem cells. Acta Biomater 6, 3978-3987 (2010).

43. Echeverri, L. F., Herrero, M. A., Lopez, J. M. \& Oleaga, G. Early stages of bone fracture healing: formation of a fibrin-collagen scaffold in the fracture hematoma. Bull Math Biol 77, 156-183 (2015).

44. De Kok, I. J., Jere, D., Padilla, R. J. \& Cooper, L. F. Evaluation of a collagen scaffold for cell-based bone repair. Int J Oral Maxillofac Implants 29, e122-129 (2014).

45. Zhou, C. et al. Biomimetic fabrication of a three-level hierarchical calcium phosphate/collagen/hydroxyapatite scaffold for bone tissue engineering. Biofabrication 6, 035013 (2014).

46. Wang, Y., Cui, F. Z., Hu, K., Zhu, X. D. \& Fan, D. D. Bone regeneration by using scaffold based on mineralized recombinant collagen. J Biomed Mater Res B Appl Biomater 86, 29-35 (2008).

47. Moshaverinia, A. et al. Encapsulated dental-derived mesenchymal stem cells in an injectable and biodegradable scaffold for applications in bone tissue engineering. J Biomed Mater Res A 101, 3285-3294 (2013).

48. Alge, D. L. et al. Donor-matched comparison of dental pulp stem cells and bone marrow-derived mesenchymal stem cells in a rat model. J Tissue Eng Regen Med 4, 73-81 (2010).

49. Stanko, P., Kaiserova, K., Altanerova, V. \& Altaner, C. Comparison of human mesenchymal stem cells derived from dental pulp, bone marrow, adipose tissue, and umbilical cord tissue by gene expression. Biomed Pap Med Fac Univ Palacky Olomouc Czech Repub 158, 373-377 (2014).

50. Kanafi, M. M., Ramesh, A., Gupta, P. K. \& Bhonde, R. R. Dental pulp stem cells immobilized in alginate microspheres for applications in bone tissue engineering. Int Endod J 47, 687-697 (2014)

51. Petridis, X., Diamanti, E., Trigas, G., Kalyvas, D. \& Kitraki, E. Bone regeneration in critical-size calvarial defects using human dental pulp cells in an extracellular matrix-based scaffold. J Craniomaxillofac Surg 43, 483-490 (2015).

52. Maraldi, T. et al. Human amniotic fluid-derived and dental pulp-derived stem cells seeded into collagen scaffold repair critical-size bone defects promoting vascularization. Stem Cell Res Ther 4, 53 (2013).

53. Furfaro, F., Ang, E. S., Lareu, R. R., Murray, K. \& Goonewardene, M. A histological and micro-CT investigation in to the effect of NGF and EGF on the periodontal, alveolar bone, root and pulpal healing of replanted molars in a rat model - a pilot study. Prog Orthod 15, 2 (2014)

\section{Acknowledgements}

This work was supported by grants from University Paris Descartes, University Paris Diderot, Fondation de la Recherche Médicale for EA2496 (DBS20131128438), Plateforme d'imagerie du Vivant Paris Descartes (FRM DGE20111123012), Fondation des Gueules Cassées for EA2496, and from the National French agency for research (ANR PulpCell 2014-2017). F. Chamieh is a post-graduate student of the European Federation of Periodontology program. 


\section{Author Contributions}

M.L.C., C.C. and G.Y.R. contributed to the design of the work. F.C., A.M.C., J.L., S.R., J.S., A.L., A.N. and G.Y.R. acquired and analyzed data. F.C., A.N., S.N.N., C.C. and G.Y.R. interpreted the data for the work. F.C., B.R.C., A.N., D.L., S.N.N., P.B., C.C. and G.Y.R. drafted or critically revised the manuscript. All authors approved of the final version of the manuscript.

\section{Additional Information}

Competing financial interests: The authors declare no competing financial interests.

How to cite this article: Chamieh, F. et al. Accelerated craniofacial bone regeneration through dense collagen gel scaffolds seeded with dental pulp stem cells. Sci. Rep. 6, 38814; doi: 10.1038/srep38814 (2016).

Publisher's note: Springer Nature remains neutral with regard to jurisdictional claims in published maps and institutional affiliations.

(c) (i) This work is licensed under a Creative Commons Attribution 4.0 International License. The images or other third party material in this article are included in the article's Creative Commons license, unless indicated otherwise in the credit line; if the material is not included under the Creative Commons license, users will need to obtain permission from the license holder to reproduce the material. To view a copy of this license, visit http://creativecommons.org/licenses/by/4.0/

(C) The Author(s) 2016 